\title{
Enrichment-free High-throughput Liquid Chromatography-Multiple-Reaction Monitoring Quantification of Cytochrome P450 Proteins in Plated Human Hepatocytes Direct from 96-Well Plates Enables Routine Protein Induction Measurements
}

\author{
John P. Savaryn, ${ }^{1}$ Ning Liu, ${ }^{1}$ Jun Sun, Junli Ma, David M. Stresser, and Gary Jenkins \\ DMPK-BA, AbbVie Inc., North Chicago, Illinois
}

Received January 7, 2020; accepted April 7, 2020

\begin{abstract}
Despite the availability of liquid chromatography (LC)-mass spectrometry (MS) methods for quantifying cytochrome P450 (P450) proteins, incorporation of $\mathrm{P} 450$ protein quantification into induction study workflows has not been widely adopted. To more readily enable P450 protein quantification in induction study workflows, DMPK research groups need a simple, robust, cost-effective, highthroughput method compatible with 96-well-plated human hepatocyte formats. Here, we provide such a methodology. Our method bypasses both microsomal enrichment and antibody-based enrichment to go directly from the plate to LC-MS/MS analysis. We use this "plate-to-peaks" approach for quantifying CYP3A4, CYP2B6, and CYP1A2, the major inducible hepatic P450s representative of pregnane $X$ receptor-, constitutive androstane receptor-, and aryl hydrocarbon receptor-mediated induction, respectively. We leveraged our induction study-aligned assay format to assess induction across mRNA, protein, and enzyme activity using known induction control compounds. As expected, results from the three methods using model inducers were broadly concordant, but the magnitude of the induction response differed. Induction of CYP3A4 using $10 \mu \mathrm{M}$
\end{abstract}

rifampicin was 12-fold for RNA, eightfold for protein, and threefold for activity; for CYP1A2 with $50 \mu \mathrm{M}$ omeprazole, induction was 30 fold for RNA, 13-fold for protein, and 17-fold for activity; for CYP2B6 with $50 \mu \mathrm{M}$ phenytoin, induction was 23-fold for RNA, twofold for protein, and fivefold for activity. Most importantly, we anticipate the relative ease of this method will enable researchers to routinely adopt $\mathbf{P 4 5 0}$ protein quantification as part of nonclinical evaluation of P450 induction.

\section{SIGNIFICANCE STATEMENT}

Current methodologies for quantifying P450 proteins by liquid chromatography (LC)-tandem mass spectrometry are either cumbersome, too costly, or both to be widely adopted into induction study workflows by the ADME research community. We present a simplified LC-MS/MS methodology for quantifying P450 proteins directly from human hepatocytes, without any form of enrichment, in 96-well induction assay plate format that should be readily adoptable by any ADME laboratory with LC-multiple-reaction monitoring capabilities.

\section{Introduction}

Drug-induced changes in cytochrome P450 (P450) expression levels can alter elimination rates of drugs that are $\mathrm{P} 450$ substrates. Both suppression and induction of $\mathrm{P} 450$ proteins may occur, but the latter is more commonly observed. Strong inducers of CYP3A4 include rifampicin, enzalutamide, and ivosidenib (Nocke-Finck et al., 1973; Gibbons et al., 2015; https://didb.druginteractioninfo. org/drug/monograph/15107/\#ddi-summary). Narrow therapeutic index drugs that are sensitive substrates of the induced $\mathrm{P} 450$ isoforms

\footnotetext{
${ }^{1}$ J.P.S. and N.L. contributed equally to this work.
}

All authors are or were employees of AbbVie at the time the work was conducted and may own AbbVie stock. N.L. is currently retired. AbbVie sponsored and funded the study; contributed to the design; participated in the collection, analysis, and interpretation of data; and participated in writing, reviewing, and approval of the final publication.

https://doi.org/10.1124/dmd.120.090480. may lose efficacy and can therefore be contraindicated when given with inducing agents.

Plated human hepatocytes have long been used as an in vitro model to evaluate P450 induction. Early versions of the assay involved measuring increases in probe substrate turnover for catalytic activity or higher levels of immunoreactive protein following a 2- to 3-day exposure to a test compound (Pichard et al., 1990). Over time, it became apparent that enzyme activity assays could be misleading in the event that the test compound was an inhibitor of the induced enzyme, as it could mask the induction response. Though presence of masking could be discerned by orthogonal Western blot analysis of the target protein, this technique is time-consuming and generally regarded as semiquantitative.

In the past decade, mRNA quantification by quantitative polymerase chain reaction (PCR) has emerged as the preferred method to evaluate in vitro induction as the measured response is unaffected by enzyme inhibition and is generally regarded to have better analytical sensitivity (Fahmi et al., 2010; FDA, 2017). However, mRNA remains a surrogate measurement for the induced protein. In addition, inherent assumptions

ABBREVIATIONS: ADME, absorption, distribution, metabolism, and excretion; CITCO, 6-(4-chlorophenyl)imidazo[2,1-b][1,3]thiazole-5-carbaldehyde O-(3,4-dichlorobenyzl)oxime; DTT, Dithiothreitol; LC, liquid chromatography; MRM, multiple-reaction monitoring; MS, mass spectrometry; P450, cytochrome P450; PCR, polymerase chain reaction; RT-PCR, real-time PCR. 
are that increases in mRNA directly correlate with an increase in functional enzyme and that processes affecting enzyme expression levels occurring post-transcription (e.g., protein stabilization, alterations in translation efficiency, rates of protein degradation) are absent.

In recent years, proteomic quantification by liquid chromatography (LC)-tandem mass spectrometry (MS) has gained wide popularity, including as part of P450 induction testing (MacLean et al., 2017). A key need for proteomic analysis in hepatocyte P450 induction studies is the measurement of an accurate baseline to serve as the denominator of a fold-induction calculation. To date, successful evaluations of induction by proteomic quantification has required a P450 protein-enrichment step, typically by preparation of microsomes. Though most laboratories pursuing this strategy have invoked ultracentrifugation methods to prepare microsomes (Jenkins et al., 2006; Langenfeld et al., 2009; Kawakami et al., 2011; Sakamoto et al., 2011; Williamson et al., 2011), $\mathrm{Xu}$ et al. (2014) demonstrated LC-MS/MS quantification of CYP3A4 from hepatocyte lysate using $\mathrm{CaCl}_{2} /$ methanol-based microsomal enrichment. In 2015, Weiß et al. (2015) circumvented the need for microsomal preparation by utilizing peptide group antibodies to enrich digested peptides for LC-MS/MS quantification of CYP3A subfamily member proteins from human hepatocytes. This technology was expanded to also quantify CYP1A2, CYP2B6, and CYP2C8 (MacLean et al., 2017) and then again to include $14 \mathrm{P} 450$ proteins in liver tissue and cultured hepatocytes in an induction experiment (Weiß et al., 2018). Though peptide group antibody-mediated enrichment is an innovative solution to avoid tedious microsomal enrichment, the dependence on antibodies, their associated cost and time to produce, and the complex nature of antibody-mediated enrichment sample preparation may be prohibitive for most researchers.

We report a novel sample preparation strategy for LC-MS/MS quantification of $\mathrm{P} 450$ proteins in plated human hepatocytes that avoids enrichment steps. The key elements of the process are minimizing sample handling, avoiding transfer steps, and eliminating the use of detergents. To quantify protein, we simply replace the culture media with a solution of ammonium bicarbonate, reducing agent (DTT), and the alkylating agent iodoacetamide. We then heat the cells to $90^{\circ} \mathrm{C}$, cool, add trypsin, digest for 3 hours, quench the reaction, and inject the supernatant for LC-MS/MS. We applied this new approach (which we term "plate-to-peaks" owing to its direct culture plate to LC-MS/MS peaks) to protein quantification of CYP3A4, CYP1A2, and CYP2B6 in hepatocyte induction studies. In this manuscript, we compare induction response from several model-inducing agents across three measurements: LC-MS/MS quantified protein, mRNA, and enzyme endpoints. In addition, we highlight two options for easing the adoption of protein quantification into routine DMPK laboratory drug-drug interaction (or other) assay formats and also assess the protein levels of CYP3A4 upon treatment with AZD1208, a compound for which in vitro induction assay results using conventional mRNA/enzyme activity assays and cutoffs largely failed to predict in vivo induction response (Jones et al., 2017).

\section{Materials and Methods}

Cell Culture and Drug Treatment. Cryopreserved primary human hepatocytes (donor ACB, sex: male) were purchased from BioIVT. Cells were thawed and plated according to the vendor's protocol. Hepatocytes were thawed in a $37^{\circ} \mathrm{C}$ water bath and decanted into prewarmed $5 \mathrm{ml} /$ vial InVitroGro CP medium (BioIVT). Cells were plated at a density of $\sim 55,000$ viable cells per well on collagen-coated 96-well culture plates. Cells were then incubated in a $5 \% \mathrm{CO}_{2}$ incubator, and the plating medium was replaced by incubation medium [Williams' Medium E $(1 \times$, no phenol red)] containing Hepatocyte Maintenance Supplements (Serum-free; Thermo Fisher) and GelTrex (Thermo Fisher) $0.35 \mathrm{mg}$ / $\mathrm{ml}$ after 4-6 hours.

After overnight stabilization, hepatocytes were treated with solvent vehicle $(0.1 \%$ DMSO) and test compounds at various concentrations with $0.1 \%$ DMSO in incubation medium for 2 days (nominal 48 hours of treatment), with media change and replenishment after 1 day. Rifampin $(10 \mu \mathrm{M})$, phenytoin $(0.1-200 \mu \mathrm{M})$, omeprazole $(0.1-200 \mu \mathrm{M})$, CITCO $(0.1 \mu \mathrm{M})$, and probenecid $(10 \mu \mathrm{M})$ were purchased from Sigma-Aldrich.

RNA Isolation. The RNA was isolated using MagMAX Express 96 RNA Isolation System from Thermo Fisher with RNA extraction kits (Thermo Fisher). Briefly, hepatocytes were washed once with $1 \times$ PBS. One hundred forty microliters of RNA lysis buffer was added to each well and mixed with the samples. The lysate was transferred to a well of the 96-well binding plate and samples were mixed with $20 \mu \mathrm{l}$ of a solution containing magnetic beads. The RNA was bound to beads, and the beads were captured on a 96-well magnetic tip manifold. The RNA sample beads were washed with wash solution. Then, the samples were treated with TURBO DNase. The RNA was rebound with beads after DNase treatment and washed twice with buffer. The beads were dried, and RNA was eluted with $50 \mu 1$ of elution buffer.

cDNA Synthesis and Real-Time PCR. cDNA synthesis followed Thermo Fisher's protocol for SuperScript VILO Master Mix (Thermo Fisher). Briefly, $4 \mu \mathrm{l}$ of SuperScript VILO Master Mix was mixed with $16 \mu \mathrm{l}$ of RNA followed by incubation at $25^{\circ} \mathrm{C}$ for 10 minutes, $42^{\circ} \mathrm{C}$ for 60 minutes, and terminated at $85^{\circ} \mathrm{C}$ for 5 minutes on a thermocycler.

Real-time PCR (RT-PCR) was performed on the ABI QuantStudio, with the following parameters: $2.5 \mu \mathrm{l}$ of each cDNA sample was pipetted into a 96-well optical reaction plate (Thermo Fisher/Applied Biosystems). Reagent mix is made using the TaqMan Fast Advanced Master Mix RT-PCR kit (Thermo Fisher/Applied Biosystems). A total of $17.5 \mu \mathrm{l}$ of each reagent mix is added to each well with 40 cycles of RT-PCR using the primer and probes from Thermo Fisher listed below:

Human CYP1A2: TaqMan Gene Expression Assay: Thermo Fisher Assay ID: Hs00167927_m1.

Human CYP2B6: TaqMan Gene Expression Assay: Thermo Fisher Assay ID: Hs03044634_m1.

Human CYP3A4: TaqMan Gene Expression Assay: Thermo Fisher Assay ID: Hs00604506_m1.

GAPDH (control to account for any variability in RNA levels): TaqMan Gene Expression Assay: Thermo Fisher Assay ID: Hs02758991_g1.

P450 Enzyme Activity Assay. After a 2-day treatment with test compounds (with media change and replenishment after 1 day), the hepatocytes were washed with incubation medium and then incubated with probe substrates as follows: $100 \mu \mathrm{M}$ of phenacetin for 60 minutes, $250 \mu \mathrm{M}$ of bupropion for 30 minutes, and $30 \mu \mathrm{M}$ of midazolam (Sigma) for 30 minutes. The enzyme activity was determined by the production of the appropriate metabolites, acetamidophenol for CYP1A2, hydroxybupropion for CYP2B6, 1'-hydroxymidazolam for CYP3A4 with stable labeled internal standards (all from Corning Life Sciences, Tewksbury, MA), ${ }^{15} \mathrm{~N}$-acetaminophen, d6-hydroxy bupropion, and ${ }^{13} \mathrm{C}_{3}-1^{\prime}$ hydroxymidazolam for CYP3A4 using area-ratio method by LC-MS

For LC-MS, samples were quenched with at least two volume equivalents of organic solvent containing internal standard, followed by centrifugation and injection of the supernatant for LC-MS/MS. Sciex Analyst 1.5 (or higher) software was used to determine peak areas for the compounds of interest. Additional LC-MS method details can be found in Table 1.

Data Analysis (mRNA and Enzyme Activity). The fold induction in P450 isoform mRNA caused by the test compounds was determined using quantitative RT-PCR and the $\Delta \Delta \mathrm{CT}$ method with the following equations: fold change $=$ $2 \Delta \Delta \mathrm{CT}$, where $\Delta \mathrm{CT}$ is the difference in threshold cycle between the target and reference genes, and $\Delta \Delta \mathrm{CT}=\Delta \mathrm{CT}$ (treated sample) $-\Delta \mathrm{CT}$ (vehicle). The fold induction in enzyme activities is determined by the metabolite production with the testing compound compared with treatment by the vehicle.

LC-Multiple-Reaction Monitoring Method Development. LC-multiplereaction monitoring (MRM) was performed on an AB SCIEX QTRAP 6500 coupled to an Agilent Technologies 1290 Infinity pump and Gerstel MultiPurpose Sampler MPS 3C autosampler. The LC column used was an Acquity UPLC CSH C18 $1.7 \mu \mathrm{m} 2.1 \times 150 \mathrm{~mm}$. The flow rate was $250 \mu \mathrm{l} / \mathrm{min}$. The column was heated to $60^{\circ} \mathrm{C}$. Mobile phase A was $0.1 \%$ formic acid in water; mobile phase B was $0.1 \%$ formic acid in acetonitrile. An optimized LC gradient was as follows: from 0 to 8 minutes, ramp mobile phase B from $2 \%$ to $50 \%$; from 8 to 8.1 minutes, ramp mobile phase B from $50 \%$ to $95 \%$; from 8.1 to 9.1 minutes, hold mobile phase B at $95 \%$; from 9.1 to 9.2 minutes, decrease mobile phase B from $95 \%$ to $2 \%$; and from 9.2 to 11.2 minutes, hold mobile phase B at $2 \%$. It should be noted 
TABLE 1

Enzyme activity assay LC-MS method details

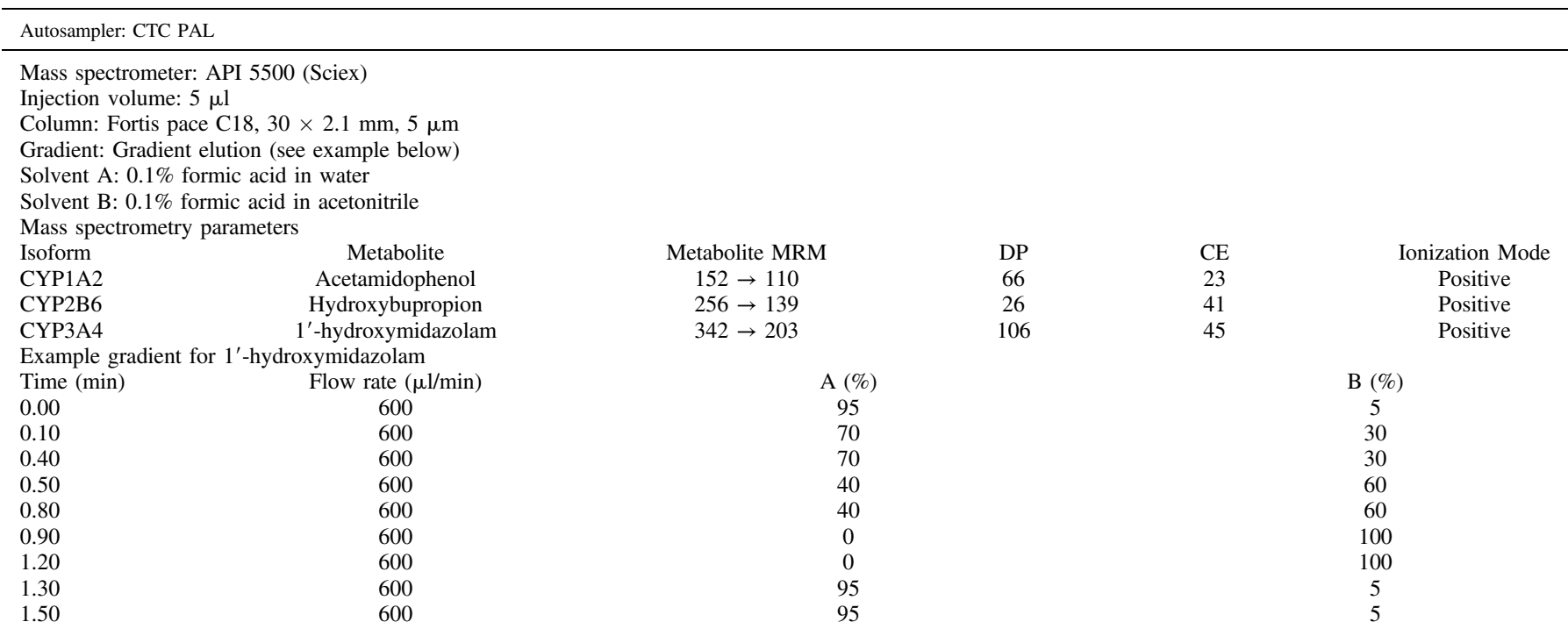

CE, collisional energy; DP, declustering potential.

that some data reported in this work were generated using slight modifications of the gradient.

Surrogate peptides were selected using a combination of in silico prediction and empirical analysis of recombinant protein digests. Skyline software was used to generate MRM methods for theoretical tryptic peptides after in silico digestion of each $\mathrm{P} 450$ protein sequence. We applied little constraint a priori on peptide selection beyond the general default Skyline parameters, opting rather to empirically test for those tryptic peptides having $2+$ or $3+$ precursors and yielding robust ionization signals. The AB SCIEX default Skyline settings for declustering potential and collisional energy were used, and transition lists were exported for empirical testing. Successive injections of recombinant protein digest were made to run the set of MRM methods covering the theoretical tryptic peptides generated from in silico digest. The MRM data were then imported into Skyline, and peptides were analyzed for their fit for use based on peak heights, peak shapes, and amount and relative abundance of fragment ions per precursor. The in silico list of theoretical tryptic peptides were evaluated for uniqueness against a tryptic digest of the human proteome. The final peptides chosen for use in analysis of biologic samples are described in Table 2. Representative electrospray source conditions are also included in Table 2.

Protein Quantification Assay. Sandwich cultured cryopreserved human hepatocytes (lot ACB, sex: male) at a density of $\sim 55,000$ viable cells per well in collagen-coated 96-well plates were treated with model inducers, experimental compound AZD1208, or other assay controls. After 48 hours of incubation with compounds in serum-free maintenance media, cell plates were washed twice with ice-cold PBS. Subsequently, $60 \mu \mathrm{l}$ of $50 \mathrm{mM}$ ammonium bicarbonate containing $5 \mathrm{mM}$ of DTT and $10 \mathrm{mM}$ of iodoacetamide (IAA) were added to each well. The plate was covered tightly using adhesive PCR plate seals (clear film, heat-resistant), heated at $90^{\circ} \mathrm{C}$ with shaking for 15 minutes, and then removed and allowed to cool to room temperature. The mixture was then digested directly in the 96-well plate by adding trypsin (MS Grade; Pierce) at an enzyme-to-protein ratio of 1:30 (w:w) to each well and incubated at $37^{\circ} \mathrm{C}$ for 3 hours. Protein concentration per well was determined initially during assay development by BCA assay after the heating step; it was omitted in subsequent studies because we always used the same fixed amount of hepatocytes (55,000 per well), and cells were always cultured for the same timeframe ( 48 hours). The reaction was stopped by adding quench solution to a final concentration of $5 \%$ acetonitrile and $0.5 \%$ trifluoroacetic acid per well. Samples were then transferred to injection plates for analysis by LC-MS/MS. A representative step-by-step protocol is described in Table 3 .

LC-MRM analysis was performed using the surrogate peptides and instrument configuration/settings described above. Relative protein quantification was performed using Skyline software. Data were imported into Skyline, and peak areas for each surrogate peptide were compared with the same surrogate peptide between treated and vehicle control samples. Data for each surrogate peptide were normalized to the peak area in vehicle control samples and are presented as fold change relative to vehicle control throughout the manuscript. We did not include an internal standard to normalize for injection-to-injection variability or protein extraction efficiency. However, cell plating efficiency in hepatocyte induction assays is typically quite uniform, and within-well sample digestion ensures efficient recovery. This was demonstrated by the use of triplicate wells for each treatment condition that showed acceptable precision.

\section{Results}

Enrichment-Free P450 Protein Quantification from Plated Human Hepatocytes in 96-Well Format by LC-MS/MS. Compared with previous LC-MS/MS methods for $\mathrm{P} 450$ protein quantification, our new method simplifies the process significantly (Fig. 1). Early methods quantified P450 proteins from liver microsomes (Alterman et al., 2005; Jenkins et al., 2006; Langenfeld et al., 2009; Kawakami et al., 2011; Sakamoto et al., 2011), a process which was commercialized in a kitbased format by AB SCIEX (Williamson et al., 2011). However, two factors impede the wide-spread adoption of such methodology: 1) microsomes need to be prepared from hepatocytes, meaning they must be cultured in low-well density format (e.g., six-well) or even dishes to ensure adequate yield of material following cell scraping; and 2) microsomal enrichment requires ultra-centrifugation, which is inherently a laborious and time-consuming process (Fig. 1A). More recent efforts have achieved $\mathrm{P} 450$ protein quantification in a form more amenable to hepatocyte induction assays by using antibody-mediated enrichment of tryptic peptides (Weiß et al., 2015, 2018; MacLean et al., 2017) (Fig. 1B). However, this technique is relatively costly, timeconsuming, and carries the added complexities of generation and use of multiple affinity reagents. To simplify the process, we tested whether $\mathrm{P} 450$ protein surrogate peptides could be detected by LC-MS/MS from plated human hepatocytes without the use of antibody-mediated peptide enrichment or microsomal enrichment (Fig. 1C). Because we complete all the sample processing in the plate and inject the supernatant directly onto LC-MS/MS (to generate peaks), we informally name our new methodology "plate-to-peaks."

Using our plate-to-peaks method, we show that basal levels of CYP3A4, CYP1A2, and CYP2B6 protein are readily measured from 
TABLE 2

Surrogate peptides and MRM transitions used for CYP3A4, CYP1A2, and CYP2B6

Bold "C" indicates carbamidomethyl Cys.

\begin{tabular}{|c|c|c|c|c|}
\hline Protein & Peptide $^{a}$ & Uniqueness & Q1 & Q3 \\
\hline \multirow[t]{4}{*}{ CYP3A4 } & GVVVMIPSYALHR & Unique & 481.27 & $843.45,746.39,659.36,422.23$ \\
\hline & LSLGGLLQPEKPVVLK & Unique & 564.35 & $909.58,683.48,555.39,455.29$ \\
\hline & ECCYSVFTNR & Also present in CYP3A5 and CYP3A7 & 588.26 & $723.38,636.35,537.28,390.21$ \\
\hline & SLL̄LPTFTSGK & Also present in CYP3A5 and CYP3A7 & 569.31 & $737.38,640.33,539.28,369.20$ \\
\hline \multirow[t]{4}{*}{ CYP1A2 } & IGSTPVLVLSR & Unique & 571.35 & $783.51,587.39,392.26,686.46$ \\
\hline & YLPNPALQR & Unique & 536.3 & $795.45,698.39,584.35,398.23$ \\
\hline & DITGALFK & Unique & 432.74 & $636.37,535.32,478.30$ \\
\hline & ASGNLIPQEK & Unique & 528.79 & $727.43,614.35,501.27$ \\
\hline \multirow[t]{4}{*}{ CYP2B6 } & IAMVDPFFR & Unique & 548.29 & $780.40,681.34,566.31$ \\
\hline & ICLGEGIAR & Unique & 494.77 & $602.33,545.30,416.26,246.16$ \\
\hline & GYGVIFANGNR & Unique & 584.3 & $678.33,531.26,460.23$ \\
\hline & ETLDPSAPK & Unique & 479.25 & $614.31,499.29,244.17,315.20$ \\
\hline \\
\hline \multicolumn{5}{|c|}{$\begin{array}{l}\text { Polarity: } \\
\text { Positive }\end{array}$} \\
\hline CUR: & \multicolumn{4}{|c|}{ 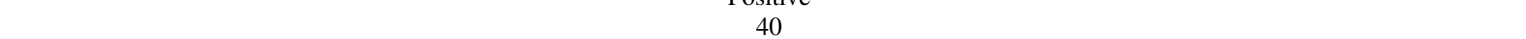 } \\
\hline IS: & \multicolumn{4}{|c|}{5500} \\
\hline TEM: & \multicolumn{4}{|c|}{450} \\
\hline GS1: & \multicolumn{4}{|c|}{60} \\
\hline GS2: & \multicolumn{4}{|c|}{70} \\
\hline CAD: & \multicolumn{4}{|c|}{ Medium } \\
\hline EP: & \multicolumn{4}{|c|}{10} \\
\hline CXP: & \multicolumn{4}{|c|}{8} \\
\hline CE: & \multicolumn{4}{|c|}{ Used Skyline predicted values } \\
\hline DP: & \multicolumn{4}{|c|}{ Used Skyline predicted values } \\
\hline
\end{tabular}

${ }^{a}$ Peptides are abbreviated to the first four N-terminal amino acids throughout the manuscript.

plated human hepatocytes in 96-well format without antibody-mediated enrichment or microsomal enrichment (Fig. 2). To empirically determine which surrogate peptides have favorable LC-MS/MS responses in MRM mode, we used Skyline software and recombinant protein digests to monitor for peak heights and LC elution profiles of all possible tryptic peptides within preset criteria (see Materials and Methods section). We pruned the transition lists per peptide based on the MRM responses and monitored for basal protein levels from plated hepatocytes that had been cultured for 48 hours. As shown in Fig. 2, we successfully detected endogenous surrogate peptides for CYP3A4 (Fig. 2A), CYP1A2 (Fig. 2B), and CYP2B6 (Fig. 2C) as evidenced by matching transition patterns compared with the positive control.

We next tested if our plate-to-peaks method was capable of quantifying $\mathrm{P} 450$ protein induction following incubation with known inducers in 96-well format. We monitored for CYP3A4, CYP1A2, and CYP2B6 protein using four surrogate peptides per protein to identify which of the four gave the maximal analytical response. We quantified induction by comparing the LC-MRM peak areas of each surrogate peptide between control and treated cells and represent the data as fold change compared with control cells (Fig. 3). Incubation with probenecid, a negative control, did not alter CYP3A4 protein levels, whereas incubation with CITCO, a constitutive androstane receptor pathway weak inducer of CYP3A4, caused up to a threefold increase in CYP3A4 protein levels (Fig. 3A). Incubation with omeprazole, an aryl hydrocarbon receptor pathway activator, caused an up to fivefold increase in CYP3A4 protein levels (Fig. 3A). As expected, incubation with increasing concentrations of rifampin, a pregnane $\mathrm{X}$ receptor pathway activator and strong inducer of CYP3A4, produced a dose-dependent increase in CYP3A4 protein levels up to 12-fold (Fig. 3B). For CYP1A2, incubation with omeprazole, an aryl hydrocarbon receptor pathway activator and strong inducer of CYP1A2, caused up to a 13-fold increase in CYP1A2 protein levels, whereas incubation with rifampin gave no increase (Fig. 3C). For CYP2B6, neither incubation with rifampin nor CITCO produced appreciable protein level changes (only

TABLE 3

P450 protein quantification sample preparation method

Slight variations on this method have also been used throughout the manuscript; the critical features of the method are represented here.

\begin{tabular}{|c|c|c|}
\hline Step & Procedure & $\begin{array}{c}\mu l \text { per } \\
\text { well }\end{array}$ \\
\hline 1 & Add $50 \mathrm{mM} \mathrm{ABC}$ & 50 \\
\hline 2 & Add $100 \mathrm{mM}$ DTT & 3 \\
\hline 3 & Add $100 \mathrm{mM}$ IAA & 7 \\
\hline 4 & Incubate covered plate at $90^{\circ} \mathrm{C}$ in dark for 15 min with $400 \mathrm{rpm}$ shaking & \\
\hline 5 & Allow plate to cool down to room temperature & \\
\hline 6 & Withdraw $10 \mu \mathrm{l} /$ well for BCA protein concentration assay & NA \\
\hline 7 & Add trypsin $(0.1 \mu \mathrm{g} / \mu \mathrm{l}$ stock concentration $)$ & 15 \\
\hline 8 & Incubate covered plate at $37^{\circ} \mathrm{C}$ for $3 \mathrm{~h}$ with $400 \mathrm{rpm}$ shaking & \\
\hline 9 & Add $10 \%$ TFA & 7 \\
\hline 10 & Add Acetonitrile & 3 \\
\hline 11 & Centrifuge at $8000 g$ & \\
\hline 12 & Add $0.1 \% \mathrm{FA}$ in water & 25 \\
\hline 13 & Inject $40 \mu \mathrm{l}$ per sample to LC-MS & \\
\hline
\end{tabular}

ABC, ammonium bicarbonate; FA, formic acid; IAA, iodoacetamide; TFA, trifluoroacetic acid. 


\section{Previous Methods}

A
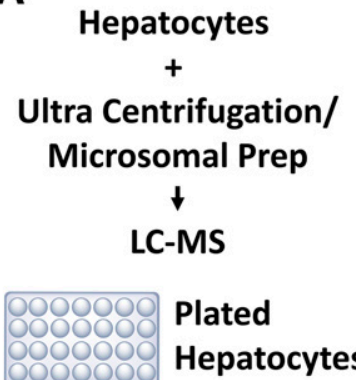

Plated

Hepatocytes
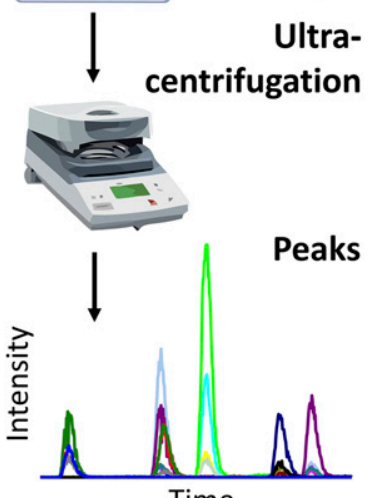

B
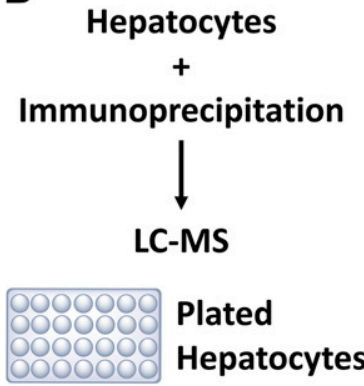

Anti-peptide antibody

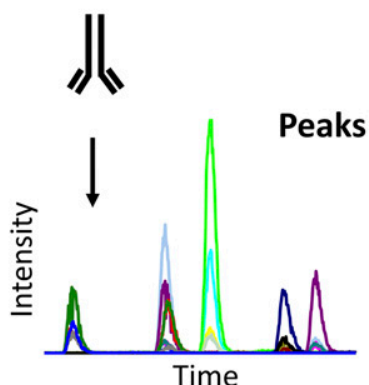

a modest $\sim$ twofold maximum for CITCO treatment), which was unexpected, as both compounds have been shown to readily induce CYP2B6 message in vitro (Faucette et al., 2007). Overall induction response profiles for these compounds across the three $\mathrm{P} 450$ isoforms evaluated are consistent with previous findings (Curi-Pedrosa et al., 1994; Rae et al., 2001).

Application of Plate-To-Peaks Protein Quantification Method to Induction Analysis of CYP3A4, CYP1A2, and CYP2B6 by RNA, Protein, and Activity. Having demonstrated the ability to monitor for

\section{Plate-to-Peaks}

C

Hepatocytes $+$

In-Plate Processing<smiles>[13CH2][13CH3]</smiles>

LC-MS

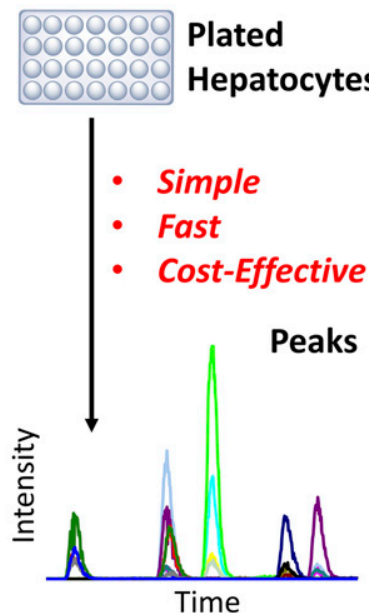

Fig. 1. Comparison of previous methods for P450 protein quantification to new plate-topeaks method.

CYP3A4, CYP1A2, and CYP2B6 protein levels by LC-MRM in 96well assay format, we next compared the induction profiles of these P450s across mRNA, protein, and enzyme activity. We incubated hepatocytes with increasing concentrations of rifampin to evaluate CYP3A4, omeprazole for CYP1A2, and phenytoin for CYP2B6 induction, and used a single surrogate peptide per $\mathrm{P} 450$ protein for quantification, the choice of which was based on our results described above and in Fig. 3. In each case, we included CITCO and probenecid as additional controls. CYP3A4 showed a dose-dependent increase in

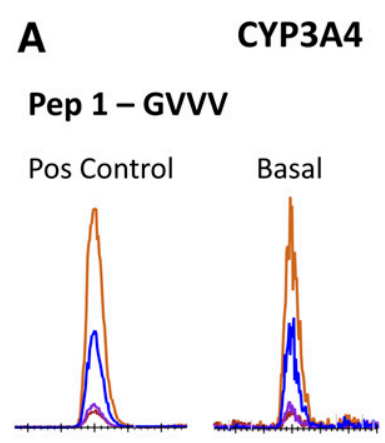

Pep 2 - LSLG

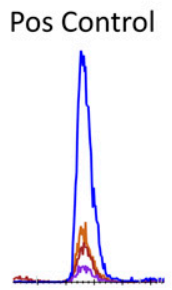

B Pep 1 - IGST

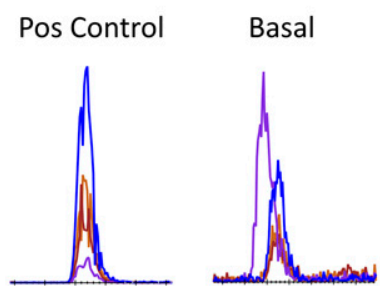

Pep 2 - YLPN

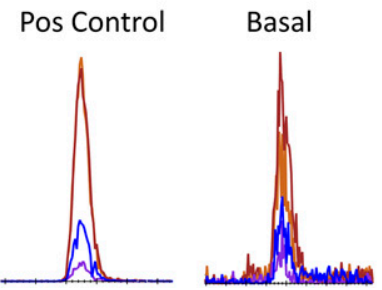

CYP1A2

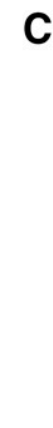

Pep 1 - IAMV

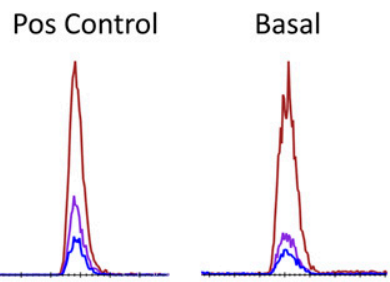

Pep 2 - ICLG

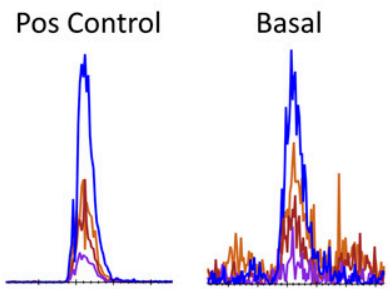

Fig. 2. LC-MRM chromatograms from plated human hepatocytes after 48 hours in culture (Basal) are compared with digestion of recombinant protein as a positive control (Pos Control) for (A) CYP3A4 surrogate peptides, (B) CYP1A2 surrogate peptides, and (C) CYP2B6 surrogate peptides. Each color represents a precursor/product ion transition belonging to the respective surrogate tryptic peptides (abbreviated as the first four $\mathrm{N}$-terminal amino acids). 


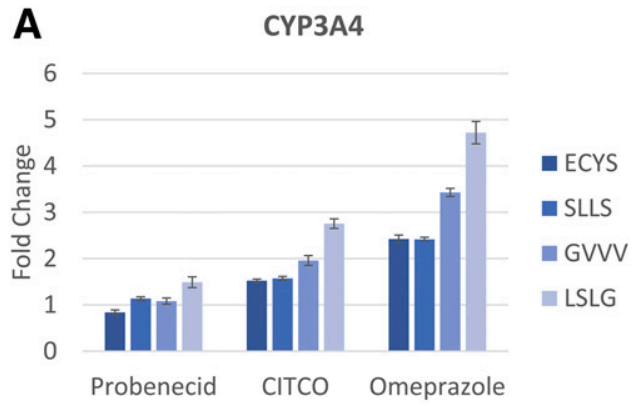

C
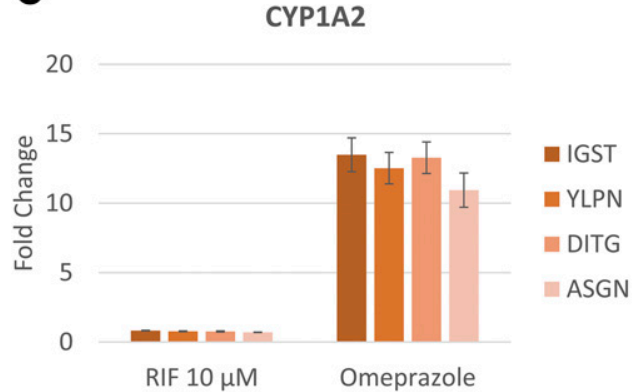

B

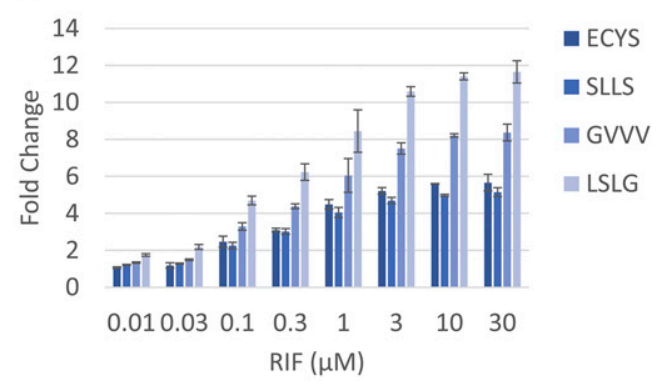

D

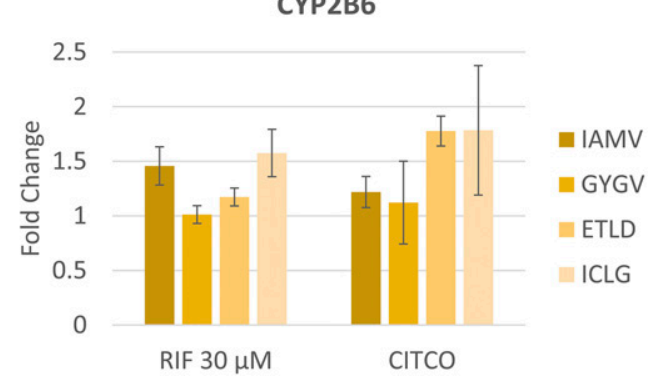

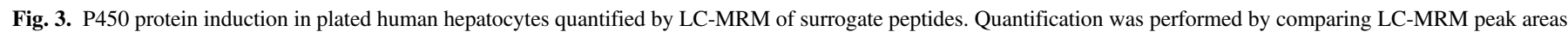

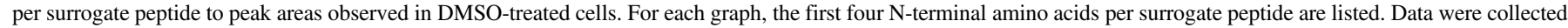

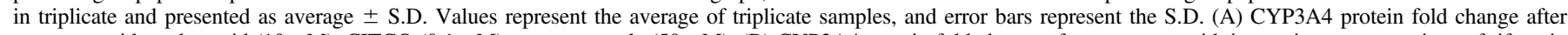

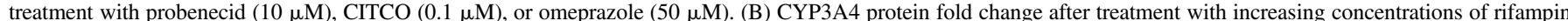

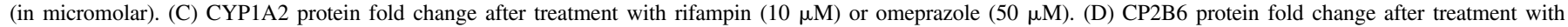
rifampin $(30 \mu \mathrm{M})$ or CITCO $(0.1 \mu \mathrm{M})$.

mRNA, protein, and activity compared with vehicle-treated control cells, with maximum effect for mRNA of 12 -fold by $10 \mu \mathrm{M}$, for protein of 9-fold by $30 \mu \mathrm{M}$, and for activity of 3-fold by $1 \mu \mathrm{M}$ (Fig. 4A). There was no induction of CYP3A4 by probenecid and modest induction by CITCO, varying in magnitude between RNA (4-fold), protein (2-fold), and activity (2-fold) (Fig. 4A). CYP1A2 showed a dose-dependent increase in mRNA, protein, and activity compared with control cells out to $50 \mu \mathrm{M}$ omeprazole treatment, after which RNA started to decline in magnitude of induction, whereas protein and activity continued to rise out to $200 \mu \mathrm{M}$, the highest concentration tested (Fig. 4B). The maximum effect for CYP1A2 was 30-fold for mRNA at $50 \mu \mathrm{M}, 23$-fold for protein at $200 \mu \mathrm{M}$, and 28-fold for activity at $200 \mu \mathrm{M}$ omeprazole (Fig. 4B). There was no induction of CYP1A2 mRNA, protein, or activity by CITCO or probenecid (Fig. 4B). CYP2B6 mRNA was induced to a maximum effect of 23 -fold by $30 \mu \mathrm{M}$ phenytoin, whereas with higher concentrations, the fold induction was either the same or decreased slightly (Fig. 4C). CYP2B6 protein was induced to a maximum effect of 2-fold by $1 \mu \mathrm{M}$ and maintained through the higher concentrations (Fig. 4C). CYP2B6 activity was induced to a maximum effect of 5-fold by $10 \mu \mathrm{M}$ and was maintained through the higher concentrations (Fig. 4C). CITCO induced CYP2B6 mRNA 15-fold, protein 2-fold, and activity 5-fold (Fig. 4C); there was no CYP2B6 induction by probenecid (Fig. 4C). Taken together, these data show the utility of our plate-topeaks processing for protein analysis in comparing induction profiles across mRNA, protein, and enzymatic activity. They also show that the induction response profile for a given P450 can vary across mRNA, protein, and activity, which is consistent with previous observations from MacLean et al. (2017).

Features of Plate-To-Peaks Protein Quantification Method Complimentary to Routine Induction Assay Formats: Direct Analysis from Fresh, Frozen, and Assay Plates. To explore options for how plate-to-peaks protein quantification could be integrated into routine P450 induction assay workflows, we tested whether target protein induction is readily quantifiable in the same plate that had been used for enzyme activity assessment just prior. Additionally, we examined whether storing plates at $-80^{\circ} \mathrm{C}$ prior to sample processing would affect protein quantification. Both options would offer benefits in the form of improved efficiency and quality (activity and protein quantification from the same plate) or introduction of a stopping point (freezing the plate for later analysis) in what otherwise could be a long process. We incubated hepatocytes with increasing concentrations of rifampin and monitored protein induction of CYP3A4 across "fresh" plates, "assay" plates (in which target protein quantification was assessed following enzyme activity assessment), and "frozen" plates, which were stored at $-80^{\circ} \mathrm{C}$ prior to thaw and extraction. All three plates showed similar induction profiles, with maximum effects of 10 -fold at the maximum rifampin concentration tested $(30 \mu \mathrm{M})$ (Fig. 5). Worth noting is that the "fresh plate" data are the average \pm S.D. of six replicate measures across two independent users (three replicates each). The relatively small error speaks to the robustness of this assay (Fig. 5). These data suggest that $\mathrm{P} 450$ protein quantification using a plate-to-peaks processing could be readily introduced into conventional induction screening workflows that typically accommodate mRNA and/or enzyme activity endpoints. When conducted, enzyme activity is assessed within wells at the end of the induction treatment. This can then be followed by cell harvest for mRNA analysis. Under this scenario, cells would no longer be available for target protein quantification, forcing the analyst to choose between using cells for mRNA or protein analysis. Therefore, we foresee replicate treatment plates being needed to accommodate all three endpoints. One could imagine scenarios in which all three endpoints could be generated within a single plate, such as a split level insert that could be removed for one workstream and plated housing cells proceed 
A

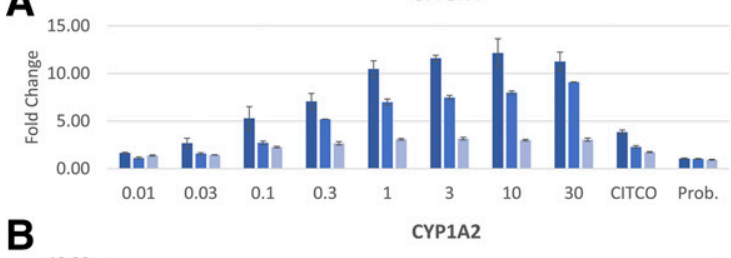

B

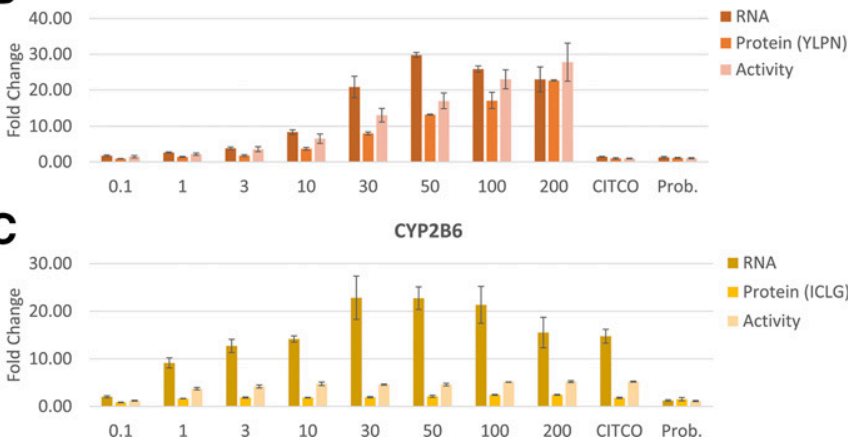

Fig. 4. Comparison of RNA, protein, and activity data for (A) CYP3A4, (B) CYP1A2, and (C) CYP2B6. In all cases, data are represented as fold change compared with levels in vehicle $(0.1 \%$ DMSO) treated controls. Data were collected in triplicate and presented as average \pm S.D. (A) Increasing concentrations of rifampin (micromolar). CITCO $=0.1 \mu \mathrm{M}$; Probenecid (Prob.) $=10 \mu \mathrm{M}$. Midazolam $(30 \mu \mathrm{M})$ was used as the substrate for activity measurements. (B) Increasing concentrations of Omeprazole (micromolar). CITCO $=0.1 \mu \mathrm{M}$; Probenecid (Prob.) $=10 \mu \mathrm{M}$. Phenacetin $(100 \mu \mathrm{M})$ was used as the substrate for activity measurements. (C) Increasing concentrations of Phenytoin (micromolar). CITCO $=0.1 \mu \mathrm{M}$; Probenecid (Prob.) $=10 \mu \mathrm{M}$. Bupropion $(250 \mu \mathrm{M})$ was used as the substrate for activity measurements.

through another workstream. The desire and effort to multiplex this assay as much as possible has been ongoing for a long time (Halladay et al., 2012).

Application of Plate-To-Peaks Method for Quantifying CYP3A4 Protein Induction by AZD1208. With the ability to quantify P450 protein induction, we evaluated AZD1208 for CYP3A4 protein induction potential in plated hepatocytes. This compound had been identified as an in vivo inducer of CYP3A4 but was not predicted as such based on in vitro data generated with HepaRG cells and human

\section{CYP3A4 (LSLG)}

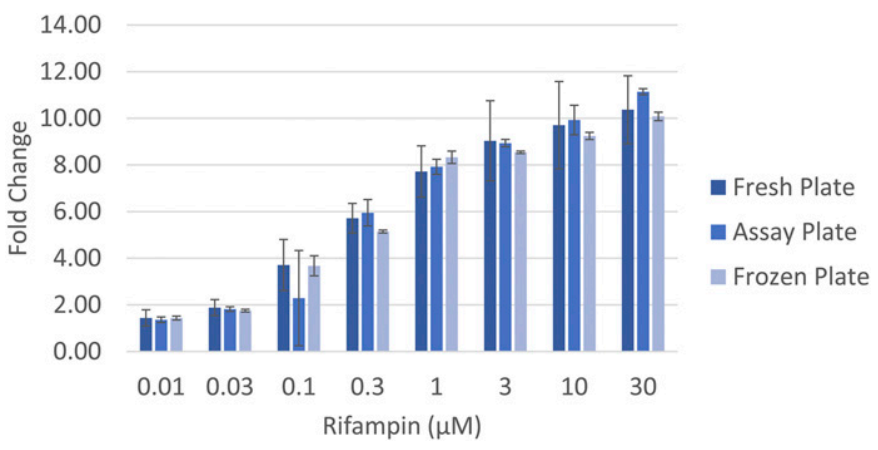

Fig. 5. Practical applications of new LC-MS/MS method for P450 protein quantification. Consistency of quantitative results, in terms of fold change compared with vehicle $(0.1 \%$ DMSO) treated cells, is compared between fresh plates, plates previously used in activity assays (assay plate), and plates frozen after incubation with rifampin but prior to sample processing (frozen plate) for CYP3A4. Note that in the $0.1 \mu \mathrm{M}$ rifampin-treated assay plate data point, one of the three replicate wells was a low outlier causing high error. Fresh plate data are the average of six replicates across two experiments run on different days and by different users (three replicates per experiment). Assay plate and frozen plate data are the average of three replicates from one experiment each. In all cases, data are presented as average \pm S.D. hepatocytes using enzyme activity and mRNA as endpoints (Jones et al., 2017). Therefore, we felt this compound would be interesting to evaluate using our approach. We treated plated human hepatocytes with increasing concentrations of AZD1208 ranging from 0.03 to $50 \mu \mathrm{M}$, covering the maximum concentrations tested by Jones et al. (2017), and monitored CYP3A4 protein induction. CYP3A4 was increased to a maximum effect of 3 -fold at $10 \mu \mathrm{M}$ AZD1208 treatment compared with control cells (Fig. 6); at lower concentrations, the increases were less in magnitude, but a clear dose-dependent increase starting at $1 \mu \mathrm{M}$ could be seen (Fig. 6); fold changes decreased at concentrations higher than $10 \mu \mathrm{M}$ (Fig. 6). The 3-fold CYP3A4 protein induction in vitro by AZD1208 shown here may be consistent with the clinical findings (Jones et al., 2017).

\section{Discussion}

We have demonstrated the ability to monitor for CYP3A4, CYP1A2, and CYP2B6 protein induction by surrogate peptide LC-MRM directly from 96-well-plated human hepatocytes without the use of antibodymediated or microsomal enrichment steps. We also compared induction across mRNA, protein, and enzymatic activity for CYP3A4, CYP1A2, and CYP2B6 using known inducers for each isoform. We elected to use a single exposure time for inducers (e.g., $2 \times 24$-hour treatments) for all three endpoints. This is because it is the format most researchers use, at least for mRNA and enzyme activity measurements. Because mRNA temporally precedes protein expression and maximal responses are not always concurrent (Zhang et al., 2010), it is an important consideration when comparing endpoint responses. Notwithstanding, induction results were broadly concordant across the three P450s analyzed and highlight the potential for facile analysis of all three major endpoints (mRNA, protein, and activity measurements) within a single experimental setting (Fig. 4, compares relative responses of mRNA, protein, and activity per P450). We anticipate such analysis will enable interrogation beyond mRNA and enzyme activity only, endpoints favored by industrial scientists and regulatory agencies since 2012 .

It has been proposed that induction of CYP2E1 [reviewed in Gonzalez (2007)] and more recently CYP2C19 (Hariparsad et al., 2017) may occur through a mechanism other than an increase in transcription, such as protein stabilization. Although CYP2E1 and CYP2C19 have important but limited roles in drug metabolism, it is tempting to speculate that major drug-metabolizing enzymes such as CYP3A4 may also exhibit induction by mechanisms in addition to transcriptional. In addition, it is conceivable that mRNA induction may be accompanied by no induction of protein, a finding which would certainly be valuable for derisking a perceived induction liability. Our method should enable rapid expansion of $\mathrm{P} 450$ protein induction analysis, which may include testing P450 proteins beyond those we quantified here. Though a global proteomics approach by nanoLC-MS/MS can readily monitor additional

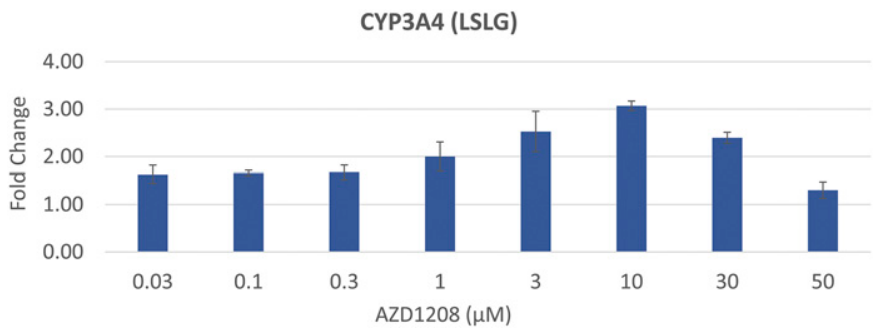

Fig. 6. Levels of $\mathrm{CP} 3 \mathrm{~A} 4$ in plated human hepatocytes following treatment with AZD1208. Quantification was performed by comparing LC-MS peak areas of the LSLG surrogate peptide between cells treated with AZD1208 at increasing concentrations and cells treated with DMSO as a control .Data were collected in triplicate and presented as average \pm S.D. 
P450 proteins, the advantage of the method we report is simplicity of process enabling quantification with analytical flow LC and conventional bioanalytical laboratory triple quadrupole MS. Over time, and as a collective body of knowledge begins to amass, we will gain a robust understanding of the diversity in mRNA, protein, and activity P450 induction profiles across a wide array of hepatocyte donors and compounds. We hope that our simplified methodology for P450 protein quantification in in vitro induction studies described here will enable such exploration. For example, we have very recently observed rifampinmediated induction of CYP3A4 protein in a second human hepatocyte donor consistent with the findings reported here (data not shown).

A common question in the field of LC-MS/MS surrogate peptide quantification is which peptide(s) should be used to represent the protein. Our data for CYP3A4 protein induction are a case in point: across four surrogate peptides for the same protein, there is up to a 2-fold difference in induction response depending on the peptide (Fig. 3, A and $\mathrm{B})$. So, which response is closer to the true induction value at the CYP3A4 protein level? At $30 \mu \mathrm{M}$ rifampin treatment, was CYP3A4 induced 6-fold or was it induced 12-fold (Fig. 3B)? Potential explanations for these differences include nonuniform digestion to peptide analyte over the range of target encountered or differences in ionization efficiencies of each peptide in relation to increased concentrations. In situations of discrepancy between surrogate peptide quantitative results, we recommend taking the highest responding peptide as closest to the true quantitative result of the protein, under the assumption that lower response peptides may be less robust in their signals because of increased susceptibility to ion suppression, variable digestion, or other phenomenon. Use of an isotopically labeled intact protein standard expressed within the biologic system of interest would test this assumption.

Our analysis raises some interesting questions. For example, why do CYP3A4 protein induction levels steadily increase to 9-fold at $30 \mu \mathrm{M}$ rifampin, whereas enzyme activity levels plateau at 3-fold beginning at $1 \mu \mathrm{M}$ of rifampin (Fig. 4A)? One explanation for this observation is that necessary components for catalytic activity (e.g., heme incorporation, expression of NADPH cytochrome $\mathrm{P} 450$ reductase, cytochrome b5) fail to track with levels of $\mathrm{P} 450$ protein expression. Because substrate concentration is well in excess of the $\mathrm{Km}$ (Michaelis constant) for midazolam 1 '-hydroxylation in our activity assay, we do not believe the observed plateau is due to substrate depletion. By contrast, CYP1A2 and CYP2B6 induction of enzyme activity is generally higher than protein levels, up to 3-fold for CYP2B6 (Fig. 4, B and C). This might be explained by choice of suboptimal peptide for quantification, yielding lower-fold induction (vide supra). Another explanation is that there is induction of enzymes in addition to CYP1A2 or CYP2B6 catalyzing probe substrate turnover.

The ultimate question for the ADME community is which measuremRNA, protein, or activity - assessed for induction in vitro best predicts induction in vivo? One could argue protein has always been the intended target for in vitro drug-drug interaction (DDI) induction assays, but because the field did not have a practical high-throughput assay, we collectively settled for mRNA and activity as surrogate measures. As reported here, protein analysis in vitro has been made possible with acceptable cost in a high-throughput format, and we therefore anticipate a growing adoption of this technique. Thus, as more laboratories quantify $\mathrm{P} 450$ protein in in vitro induction studies, it will be interesting to learn whether a single endpoint or combinations of the three will yield best predictions. Evaluation of all three endpoints may also yield insight into compound-dependent mechanism of induction, such as relative roles of transcriptional regulation and protein stabilization.

To speed the adoption of our plate-to-peaks methodology into routine in vitro workflows across the industry, we showed how it could be integrated into current workflows in one of three ways: use a fresh plate and process right away, use a fresh plate and freeze it for later processing, or use the activity assay plate after taking the supernatant for activity assessment. In our hands, each of these approaches yielded the same induction results (Fig. 5). The value of having options like this for P450 protein quantification is that it allows for integration of protein quantification into current workflows for in vitro induction by ADME laboratories without an overhaul to their current process. Thus, induction assays can carry on as usual while the same laboratories begin to explore P450 protein quantification in addition. If confronted with an already long day for an mRNA-based induction assay, now the analytical scientist can simply incubate an extra plate of hepatocytes with compound and freeze it for protein quantification later; the mRNA assay carries on unimpeded. If workflows cannot readily accommodate additional plates for compound incubation, the same plate used for assessing enzyme activity can be also used for protein quantification, i.e., two assays from the same plate. As more ADME laboratories amass their own results for $\mathrm{P} 450$ induction by protein analysis, we might collectively decide that $\mathrm{P} 450$ protein is the better endpoint for in vitro assays and thus experience a shift toward protein quantification as the primary assay.

Upon evaluating AZD1208 induction potential for CYP3A4 protein, we observed two results of note: 1) CYP3A4 protein induction reached 3-fold at a similar concentration of compound to that which produced 3-fold mRNA induction as previously reported (Fig. 6) (Jones et al., 2017), and 2) at higher concentrations of compound, CYP3A4 protein induction started to decrease (Fig. 6). It would be interesting to see how consistent the 3-fold CYP3A4 protein induction is across multiple hepatocyte donors. The decrease in the magnitude of CYP3A4 induction at concentrations higher than $10 \mu \mathrm{M}$ could be explained by cell death and the dead cells being washed away during the early steps of the process. If that were true, accounting directly for cell death in the protein quantification assay would be needed for accurate interpretation of induction on a per-cell basis. We suggest the inclusion of a panel of common housekeeping genes in MRM assays to serve as internal controls to which P450 protein induction can be normalized to-a solution that could be accommodated within our plate-to-peaks process with no negative impact owing to fast LC-MS/MS duty cycles of triple quadrupole mass spectrometers and their inherent ability to multiplex and monitor multiple peptides per injection. A final thought is in the power of orthogonal methodology to increase the confidence in results seen. In the case of AZD1208, in vitro mRNA results did pick up a threefold induction, but without any other method to confirm this relatively low response, perhaps this was disregarded as not significant enough or too low. With the studies described in this manuscript, the addition of protein measures of induction can now be performed to inform on induction potential and to highlight potential disconnects for further interrogation on the potentially complex mechanisms behind $\mathrm{P} 450$ induction.

\section{Acknowledgments}

The authors would like to acknowledge Hardikkumar Patel for bioanalytical support quantifying metabolite production in the cytochrome P450 enzyme activity assays.

\section{Authorship Contributions}

Participated in research design: Savaryn, Liu, Stresser, Jenkins.

Conducted experiments: Liu, Sun, Ma.

Contributed new reagents or analytic tools: Liu.

Performed data analysis: Savaryn, Liu, Sun, Ma.

Wrote or contributed to the writing of the manuscript: Savaryn, Liu, Stresser, Jenkins. 


\section{References}

Alterman MA, Kornilayev B, Duzhak T, and Yakovlev D (2005) Quantitative analysis of cytochrome p450 isozymes by means of unique isozyme-specific tryptic peptides: a proteomic approach. Drug Metab Dispos 33:1399-1407.

Curi-Pedrosa R, Daujat M, Pichard L, Ourlin JC, Clair P, Gervot L, Lesca P, Domergue J, Joyeux H, Fourtanier G, et al. (1994) Omeprazole and lansoprazole are mixed inducers of CYP1A and CYP3A in human hepatocytes in primary culture. J Pharmacol Exp Ther 269: 384-392.

Fahmi OA, Kish M, Boldt S, and Obach RS (2010) Cytochrome P450 3A4 mRNA is a more reliable marker than CYP3A4 activity for detecting pregnane $\mathrm{X}$ receptor-activated induction of drug-metabolizing enzymes. Drug Metab Dispos 38:1605-1611.

Faucette SR, Zhang TC, Moore R, Sueyoshi T, Omiecinski CJ, LeCluyse EL, Negishi M, and Wang $H$ (2007) Relative activation of human pregnane $X$ receptor versus constitutive androstane receptor defines distinct classes of CYP2B6 and CYP3A4 inducers. J Pharmacol Exp Ther 320:72-80.

FDA (2017) In vitro metabolism- and transporter- mediated drug-drug Interaction studies: guidance for industry. Draft Guidance.

Gibbons JA, de Vries M, Krauwinkel W, Ohtsu Y, Noukens J, van der Walt JS, Mol R, Mordenti J, and Ouatas T (2015) Pharmacokinetic drug interaction studies with enzalutamide. Clin Pharmacokinet 54:1057-1069.

Gonzalez FJ (2007) The 2006 Bernard B. Brodie Award Lecture. Cyp2e1. Drug Metab Dispos 35: $1-8$.

Halladay JS, Wong S, Khojasteh SC, and Grepper S (2012) An 'all-inclusive' 96-well cytochrome P450 induction method: measuring enzyme activity, mRNA levels, protein levels, and cytotoxicity from one well using cryopreserved human hepatocytes. J Pharmacol Toxicol Methods 66: $270-275$

Hariparsad N, Ramsden D, Palamanda J, Dekeyser JG, Fahmi OA, Kenny JR, Einolf H, Mohutsky M, Pardon M, Siu YA, et al. (2017) Considerations from the IQ induction working group in response to drug-drug interaction guidance from regulatory agencies: focus on downregulation, CYP2C induction, and CYP2B6 positive control. Drug Metab Dispos 45:1049-1059.

Jenkins RE, Kitteringham NR, Hunter CL, Webb S, Hunt TJ, Elsby R, Watson RB, Williams D, Pennington SR, and Park BK (2006) Relative and absolute quantitative expression profiling of cytochromes P450 using isotope-coded affinity tags. Proteomics 6:1934-1947.

Jones BC, Rollison H, Johansson S, Kanebratt KP, Lambert C, Vishwanathan K, and Andersson TB (2017) Managing the risk of CYP3A induction in drug development: a strategic approach. Drug Metab Dispos 45:35-41.

Kawakami H, Ohtsuki S, Kamiie J, Suzuki T, Abe T, and Terasaki T (2011) Simultaneous absolute quantification of 11 cytochrome $\mathrm{P} 450$ isoforms in human liver microsomes by liquid chromatography tandem mass spectrometry with in silico target peptide selection. J Pharm Sci 100 $341-352$.
Langenfeld E, Zanger UM, Jung K, Meyer HE, and Marcus K (2009) Mass spectrometry-based absolute quantification of microsomal cytochrome P450 2D6 in human liver. Proteomics 9: 2313-2323.

MacLean C, Weiß F, Poetz O, and Ebner T (2017) Concept: the use of targeted immunoaffinity proteomics for routine assessment of in vitro enzyme induction. J Pharm Sci 106:3453-3457.

Nocke-Finck L, Breuer H, and Reimers D (1973) [Effects of rifampicin on the menstrual cycle and on oestrogen excretion in patients taking oral contraceptives]. Dtsch Med Wochenschr 98: $1521-1523$.

Pichard L, Fabre I, Fabre G, Domergue J, Saint Aubert B, Mourad G, and Maurel P (1990) Cyclosporin A drug interactions. Screening for inducers and inhibitors of cytochrome P-450 (cyclosporin A oxidase) in primary cultures of human hepatocytes and in liver microsomes. Drug Metab Dispos 18:595-606.

Rae JM, Johnson MD, Lippman ME, and Flockhart DA (2001) Rifampin is a selective, pleiotropic inducer of drug metabolism genes in human hepatocytes: studies with cDNA and oligonucleotide expression arrays. J Pharmacol Exp Ther 299:849-857.

Sakamoto A, Matsumaru T, Ishiguro N, Schaefer O, Ohtsuki S, Inoue T, Kawakami H, and Terasaki T (2011) Reliability and robustness of simultaneous absolute quantification of drug transporters, cytochrome P450 enzymes, and Udp-glucuronosyltransferases in human liver tissue by multiplexed MRM/selected reaction monitoring mode tandem mass spectrometry with nanoliquid chromatography. J Pharm Sci 100:4037-4043.

Weiß F, Hammer HS, Klein K, Planatscher H, Zanger UM, Norén A, Wegler C, Artursson P, Joos TO, and Poetz O (2018) Direct quantification of cytochromes P450 and drug transporters-A rapid, targeted mass spectrometry-based immunoassay panel for tissues and cell culture lysates. Drug Metab Dispos 46:387-396.

Weiß F, Schnabel A, Planatscher H, van den Berg BH, Serschnitzki B, Nuessler AK, Thasler WE, Weiss TS, Reuss M, Stoll D, et al. (2015) Indirect protein quantification of drug-transforming enzymes using peptide group-specific immunoaffinity enrichment and mass spectrometry. Sci Rep 5:8759.

Williamson BL, Purkayastha S, Hunter CL, Nuwaysir L, Hill J, Easterwood L, and Hill J (2011) Quantitative protein determination for CYP induction via LC-MS/MS. Proteomics 11:33-41.

Xu L, Ma B, Yu S, Xia C, and Wu JT (2014) The use of a rapid MS-based method for the quantification of the CYP 3A4 protein directly from hepatocyte cell lysate for CYP induction studies. Bioanalysis 6:3271-3282.

Zhang JG, Ho T, Callendrello AL, Crespi CL, and Stresser DM (2010) A multi-endpoint evaluation of cytochrome P450 1A2, 2B6 and 3A4 induction response in human hepatocyte cultures after treatment with $\beta$-naphthoflavone, phenobarbital and rifampicin. Drug Metab Lett 4:185-194.

Address correspondence to: Dr. Gary Jenkins, AbbVie Inc, $1 \mathrm{~N}$ Waukegan Rd. AP9, North Chicago, IL 60064. E-mail: gary.jenkins@abbvie.com 